\title{
Stochastic methods in reliability and risk management
}

\author{
Lirong Cui • Haijun Li • Susan H. Xu
}

Published online: 23 October 2013

(C) Springer Science+Business Media New York 2013

This volume focuses on stochastic methods developed for reliability modeling and risk analysis. Reliability theory and risk analysis have been closely related, and stochastic methods that are commonly used in both fields serve as a natural conduit through which useful concepts and best practices can migrate from one field to the other. This special volume highlights this convergence of reliability modeling and risk analysis, that forms the core of the science of analyzing hazards and its applications to various fields.

Most of papers appearing in this volume were selected from the presentations given at the 7th International Conference on Mathematical Methods in Reliability (MMR2011), held in Beijing, China, June 20-24, 2011. The conference was co-chaired by Yiming Wei (Beijing Institute of Technology, China), N. Balakrishnan (McMaster, Canada), and N. Limnios (UTC, France). The papers have been significantly extended from the conference presentations and all papers have been refereed according to the standards of Annals of Operations Research.

The MMR2011 is the seventh in a series of international conferences on Mathematical Methods in Reliability. Since 1997, when the first of MMR conferences was held in Bucharest, Romania, the MMR has become a major international gathering of researchers from the international reliability community. The MMR conferences intend to serve as a forum for discussing fundamental issues and recent advances in reliability theory and risk analysis methods, as well as their applications, and have attracted an increasing number of participants from every continent in the world. The Beijing conference focused on all aspects

L. Cui

Beijing Institute of Technology, Beijing, China

e-mail: lih@math.wsu.edu

H. Li (凶)

Washington State University, Pullman, WA, USA

e-mail: lih@math.wsu.edu

S.H. Xu

Pennsylvania State University, College Park, PA, USA 
of mathematical methods in reliability and risk, and their applications in various engineering fields. The talks on related topics such as quantitative risk management, decision analysis under uncertainty, and their applications in areas such as supply chains management, sustainable energy systems and financial engineering were presented.

We thank the MMR2011 host institution, Beijing Institute of Technology, for making this conference possible. Special thanks go to Professor Endre Boros, Editor-in-Chief of AOR for giving us the opportunity to organize and edit this special volume, and to the reviewers who made tireless efforts to improve the quality and presentation of the selected papers.

Guest Editors 